\title{
Spontaneous resolution of labial adhesions in pre-pubertal girls
}

\author{
Original article.
}

Jean E Norris, Charlotte V Elder, Angela M Dunford, Deepti Rampal, Charleen Cheung, Sonia R Grover

The Royal Children's Hospital, 50 Flemington Rd, Parkville, 3052, Victoria

Corresponding author:Prof Sonia Grover,

Dept Gynaecology,

The Royal Children's Hospital,

50 Flemington $\mathrm{Rd}$,

Parkville 3052

sonia.grover@rch.org.au

$+61393455522$

This is the author manuscript accepted for publication and has undergone full peer review but has not been through the copyediting, typesetting, pagination and proofreading process, which may lead to differences between this version and the Version of Record. Please cite this article as doi: 10.1111/jpc.13847

This article is protected by copyright. All rights reserved. 


\title{
Spontaneous resolution of labial adhesions in pre-pubertal girls
}

\begin{abstract}
Aims: This study sought to observe the rate and timing of spontaneous resolution of labial adhesions that had been diagnosed in prepubertal girls. The secondary aim was to evaluate the conservative approach to management of labial adhesions that has been advocated by the gynaecology department, by assessing the rates of concurrent uro-gynaecological symptoms in this population, as well as parent satisfaction with their child's management.
\end{abstract}

Methods: A retrospective chart review of all patients diagnosed with labial adhesions in the department of paediatric and adolescent gynaecology between 2000 and 2017 was performed. Patients and their parents (depending on the age of the patient at the time of this study) were then invited to participate in follow-up surveys and questionnaires, and clinical examination.

Results: Of 148 girls identified, the median age of the follow up participants( $(n=45)$ was 6.1years (2.6-27.2years), compared to that of the entire cohort of 7.4 years(1-27y). After a median follow-up period of 2.6 years (0.4 - 20.7 years), 40\%(18/45) of girls reported and/or had findings that supported resolution of labial adhesions without treatment. Two parents (4\%) sought treatment elsewhere after their appointment. A history of UTI was reported in $30 \%(14 / 45)$ of patients and 16\% (7/45) had a known history of vulvovaginitis.

Conclusions: Our findings support the natural history of spontaneous resolution of labial adhesions. Concurrent uro-gynaecological conditions that developed were successfully treated according to standard treatments. A conservative approach to management of labial adhesions is associated with very low rates of parental concern and intervention.

\section{Key words: prepubertal, labial adhesions, labial fusion, labial agglutination,} management 


\section{What is already known}

- Labial adhesions are a common acquired gynaecological condition in prepubertal girls

- When labial adhesions are deemed to be symptomatic, different treatment modalities have been advocated and compared for their efficacy

- Little attention has been paid to the spontaneous resolution of labial adhesions despite this being recognised

\section{What this paper adds}

A conservative management of labial adhesions is advocated for the following reasons:

- It does not cause adverse clinical outcomes

- It avoids unnecessary and potentially distressing interventions

- Given adequate explanation, it is associated with resolution of parental distress and complaints of urinary tract and vulvo-vaginitis symptoms

\section{Introduction}

Labial adhesions, also known as labial agglutination or labial fusion, are a common acquired gynaecological finding in pre-pubertal girls ${ }^{1}$. They rarely cause problems and tend to disappear without intervention prior to puberty ${ }^{2-4}$. However, disagreement exists about management of girls who present with minor uro-genital symptoms or whose parents request treatment ${ }^{5}$. Some doctors also continue to treat in the absence of symptoms or parental concern ${ }^{6-8}$. There is a large body of literature comparing different treatment modalities for labial adhesions, with widely variable results ${ }^{1,6,9-11}$. However, little research addresses whether the condition actually causes the symptoms that have been attributed to it and the optimal management when symptoms or concerns are present. 
Labial adhesions are not present at birth, occurring most commonly between six months and two years of age ${ }^{12}$. They are thought to develop after local irritation denudes the thin, non-oestrogenised labial skin, and re-epithelialisation of the closely apposed labia minora results in midline adherence ${ }^{4}$. Urinary retention as a consequence of labial adhesions is rare, with one study reporting $3 / 289$ girls cases in 15 years ${ }^{13}$. The etiology of postpubertal labial adhesions is substantially different, most relating to childbirth trauma ${ }^{14}$, postmenopausal atrophic changes ${ }^{15}$ or inflammatory dermatological conditions ${ }^{16}$.

Labial adhesions are thought to be more common than outpatient presentations suggest ${ }^{17}$, as examinations are rarely performed on pre-pubertal girls after infancy, except when indicated by symptoms. As a result, adhesions are discovered at the time of presentation with local irritation, voiding difficulties, or urinary tract symptoms. Asymptomatic adhesions may be detected by maternal child health nurses or parents ${ }^{18}$. Girls may then be referred to paediatricians or paediatric gynaecologists for a variety of reasons, including parental concern ${ }^{1,7,9}$, suspected vaginal agenesis ${ }^{18}$, imperforate hymen ${ }^{4}$, local symptoms or the doctor's perception that labial adhesions require specialist management. Reported estimates of the incidence of labial adhesions in pre-pubertal girls, both symptomatic and asymptomatic, range from $1.8 \%{ }^{12}-38.9 \%{ }^{19}$. This difference may be attributable to cohort differences, with some studies recruiting patients specifically for genital examinations ${ }^{19}$, ${ }^{20}$, and others recruiting for whole body examinations ${ }^{12}$. The examination position effects the rate of detection of labial adhesions, with frog leg and knee chest positions giving rates of $38.9 \%$ and $13.9 \%$ respectively ${ }^{19}$. Additionally, the age of girls studied impacts on the reported rate $^{12}$.

Treatment for labial adhesions is controversial, although most current guidelines agree that it is unnecessary to treat 'asymptomatic' adhesions ${ }^{2-4}$. However, most recommend topical treatment with oestrogens or betamethasone creams in those girls with 'symptoms' such as 
UTI and vulvovaginitis ${ }^{2-5}$. This is based on the presumption that these conditions are secondary to labial adhesions rather than simply concurrent, although this relationship has not been explored or confirmed ${ }^{21,22}$. Furthermore, the proposed aetiology of labial adhesions, that is, inflammation of the labial epithelium with subsequent adherence, overlaps considerably with the aetiologies of these other conditions. Another common reason for girls to receive treatment for their adhesions is to alleviate parental concern ${ }^{1,7,9}$ due to the perception that their daughter's genitalia look 'abnormal ${ }^{7}$ or the desire to 'take action' to resolve the condition?.

Topical oestrogen cream is commonly utilised with reported efficacy rates of 15.5$100 \%^{11,23}$, although recurrence rates up to $34 \%$ have been reported ${ }^{6}$. Topical steroids are also used with similar efficacy ${ }^{11}$. Manual separation by lateral traction and surgical separation are usually reserved for cases not responsive to oestrogen treatment, or for 'symptomatic' adhesions ${ }^{1}$, although some clinicians recommend lateral traction as first line management ${ }^{18}$. Recorded rates of recurrence for manual separation and surgical separation vary from $0^{7}-76 \%{ }^{18}$ and $0^{24}-40 \%{ }^{25}$ respectively. In view of the recurrence rates for both manual separation and topical treatment, it has been suggested that risks associated with repeated treatments outweigh the benefits ${ }^{26}$. Risks associated with manual separation include the potential for pain and distress, and the prolonged, daily application of creams to the child's labia may cause confusion between care and intimacy. Topical oestrogen use can cause systemic absorption and breast budding and overuse of topical steroids can cause local skin thinning. Both surgical and medical approaches may cause unwarranted concern about the child's external genitalia in the parent and/or the child ${ }^{26}$. There has been only one small, prospective, observational study investigating the rate of spontaneous resolution ${ }^{27}$ which reported adhesion resolution in $100 \%(\mathrm{n}=10)$ within 18 months of diagnosis.

At this institution, the gynaecology department has advocated a conservative approach for 20 years. This approach arose from a combination of adolescent and adult 
gynaecological clinical experience and a clinical audit. Adolescent and adult women never have unexplained labial adhesions, suggesting a natural history of spontaneous resolution. An audit (Y Anbazhagan, SRGrover, unpublished data, 2000) found that manual and surgical separation in mostly asymptomatic girls was associated with high recurrence rates and repeat interventions, as well as high levels of distress and anxiety for parents and patients. The focus of management has been to provide reassurance to parents, primarily that labial adhesions are common, usually do not cause any problems and are exceedingly rare after puberty. If the child has any concurrent conditions such as vulvovaginitis or UTI, standard advice for managing these conditions is provided. If the child experiences post-void dribbling, simple toileting advice is offered. It was anticipated that once parents understood that the condition has no effect on their daughter's future fertility and would most likely resolve before puberty, that they would not return to the clinic or seek treatment for their daughter.

This is an audit of this conservative management of labial adhesions (with institutional Research and Ethics approval HREC 36361A). The primary aim was to determine the rate of spontaneous resolution of labial adhesions. The secondary aims were to determine whether

there were any ongoing symptoms associated with this conservative approach, and review parental satisfaction with their child's treatment and the information they received.

\section{Methods}

Patients identified with labial adhesions through the gynaecology clinic database and seen between January 2000-February 2017 were traced and invited to participate. Girls with dermatological conditions were excluded.

Medical records were reviewed to determine their age at presentation, referral indication, symptoms, extent of adhesions at initial assessment, any treatment provided and any subsequent review.

The extent of labial adhesions was retrospectively classified on a scale of 1 to 6 , based on 
the proportion of the fused labia minora reported in the notes:

[1]Open(=complete resolution); [2]<25\% fused; [3]25-49\% fused; [4]50-74\% fused; [5]75$98 \%$ fused; [6]99\% fused. Adhesions were sometimes described by size of the opening (in millimetres); the proportion of adherent labia, or using terms such as 'small opening', 'pinhole opening', and 'complete fusion'. 'The latter two were classified as 99\% fused, whereas a 'small opening' was defined as $75-98 \%$ fused.

For girls aged $<18$ years, parents were contacted. Of those seen within the last 2 years, a letter of invitation to participate in the study was sent, whereas those seen $>2$ years ago were sent a tracing letter. Girls aged $>18$ years were sent tracing letters. For girls $<16 y e a r s$, the invitation included the option of visiting the hospital for a genital examination to assess their adhesions. If no response had occurred within two weeks, telephone contact was attempted to participants or their parents on their last known telephone number.

Parents of girls $<16$ years were asked to complete a questionnaire to determine whether their daughter had experienced any urogenital symptoms that might be attributable to labial adhesions. Parental satisfaction with conservative management was also assessed. Parents who were willing to participate but unable to bring their daughter to clinic during the study period completed the questionnaire over the phone and were asked about persistence or resolution of their child's adhesions. Young women aged $>16$ years were asked to complete a survey to determine whether they could recall any urogenital symptoms as a child, and whether they now used tampons or had been sexually active. Labial adhesions were considered to have resolved if they were using tampons or having sex.

For girls who were examined, where adhesions were present, the examiner (a trained paediatric and adolescent gynaecologist) measured the opening in millimetres and estimated the proportion of the labia that remained fused. The presence of any vulval inflammation was documented.

The extent of adhesions at the time of consultation and diagnosis was compared to the 
findings at follow-up, to quantify how the labial adhesions had changed over time. As the extent of initial adhesions was determined retrospectively, accurate comparison was not always possible, allowing only for whether resolution had occurred by follow-up.

Secondary outcomes related to the identification of urogenital problems associated with labial adhesions and how often parents sought further advice from other medical professionals. If parents took their child elsewhere for treatment, this was interpreted as evidence that they had been dissatisfied with their child's initial management, or that the advice had not allayed their concerns. Hospital research and ethics approval for this project and patient/ parent consent obtained.

\section{$\underline{\text { Results }}$}

Of the 148 girls who were identified with labial adhesions from the database, no contact could be established with 100 . Of the 47 girls with whom contact was established, two girls were managed with topical oestrogen. One, whose labial adhesions had been diagnosed as a baby and managed conservatively, was prescribed topical oestrogen when she presented at 12 years, prepubertal, complaining of urinary stream disturbance, with urine splashing onto her thighs. The labial adhesions were thought to be causing the flow disturbance. Application of topical oestrogen for 6 weeks resulted in resolution of adhesions but urine spraying persisted. The second, at age 3 years, was prescribed topical oestrogen because she was exhibiting genital self-touching which was attributed to her labial adhesions. Adhesions resolution occurred, but the behaviour persisted. She was subsequently diagnosed with a sensory processing disorder, which may have contributed to the initial symptoms. In both cases, treatment resolved the adhesions but not the symptoms, suggesting that the symptoms were coincidental, rather than a consequence of the adhesions. Our audit also found one 15month-old girl seen in the emergency department with acute urinary retention. Her adhesions were separated with lateral traction under 
anaesthesia, with no recurrence in the subsequent 6 months with ongoing application of vaseline. This represents the only case of acute retention seen by this department in the hospital. The medical notes audit revealed five girls with resolution of adhesions noted on clinic review.

Questionnaires were completed by 42 parents and 20 girls were examined. Three young women completed online surveys. The median age of study participants was 6.1years(2.627.2years), compared to that of the entire cohort, 7.4 years(1-27y) (see Figure 2). Initial adhesions of 6 girls could not be classified, and thus the change in the extent of adhesions over time could not be determined for these patients. For the 22 girls who were not examined, some parents reported adhesion resolution, whilst four were unsure. We could classify only some of these patients' adhesions based on their parent's description.

After a median follow-up period of 2.6 years(0.4-20.7 years), 40\%(18/45) of girls reported and/or had findings that supported resolution of labial adhesions without treatment(see Figure 3). Their median age at follow-up was 7.9 years. Of girls examined, $40 \%(8 / 20)$ had complete resolution of adhesions. The five girls with resolution seen in clinic but not the study are not included to avoid bias.

Of girls who still had adhesions (mean age 5.0 years), all were prepubertal. Where it was possible to assess the change in adhesions over time, $40 \%(6 / 15)$ of girls with persistent adhesions had a reduction in the extent of their adhesions(Figure 4 ).

Of parents who completed questionnaires over the telephone, $32 \%(7 / 22)$ reported adhesions resolution, $50 \%(11 / 22)$ reported persistence of adhesions and 14\%(4/22) were unsure. The median age of patients with persistent adhesions was 4.7 years. Of the three respondents aged >16(ages 18, 27 and 24), all had used tampons, engaged in penetrative vaginal intercourse and had no gynaecological concerns.

As part of routine clinical care, parents had been encouraged to return for review if they had ongoing concerns. Of the 47 patients followed up, only 6 patients had returned for follow-up appointments. Of the total cohort $(\mathrm{n}=147), 17$ patients had more than one appointment, 
including 5 who were found to have complete spontaneous resolution. Two participating parents $(2 / 42)$ had sought treatment outside after their initial appointment. One mother was having issues with her daughter's toilet training and was prescribed oestrogen cream by her general practitioner. The other had seen a paediatrician who had recommended oestrogen cream, prior to the gynaecology appointment. Although the child was asymptomatic, topical oestrogen cream was utilised despite gynaecology service advice. A side-effect of vulval irritation occurred secondary to the cream and was discontinued. Of the 47 participants, 12 (26\%) had received treatment before referral. Six had used topical oestrogens, two topical steroids and one had been prescribed both. Two girls had their adhesions separated manually and one surgically - all three described the procedure as distressing (see accounts, Box 1). Of the 148 patients seen in the Gynaecology department, it was documented that 21 had received prior treatment.

A history of UTI was reported in 30\% (14/45) of patients and 16\% (7/45) had a documented history of vulvovaginitis. The parents of girls who had experienced urinary stream disturbance considered it an inconvenience, but were satisfied with simple strategies recommended for draining residual urine.

\section{Discussion}

Previous studies on labial adhesion management have been conducted on cohorts with a

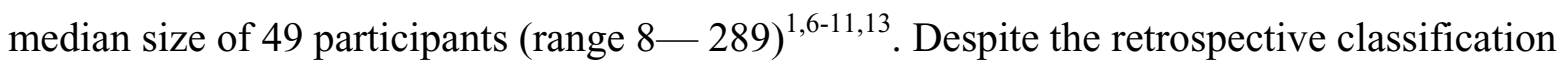
of the extent of labial fusion, the classification based on the proportion fused rather than an exact measurement of the opening allowed for comparison of labial adhesions at two different ages when labial size itself will have changed. There was a high rate of spontaneous resolution of labial adhesions in the follow-up cohort.

The low rate of follow-up examinations reflects the sensitive nature of the examination with some parents reluctant to bring daughters to clinic as their daughters were unaware of their childhood diagnosis, and others not wanting to generate anxiety about their gynaecological 
health. It was also common for parents to think older daughters would be more distressed by the examination than younger girls. Parents of school-aged children were reluctant to take their child out of school, or take time off work to attend the hospital for examination. This in itself may be reassuring, as it would be expected that parents with concerns about their child's uro-gynaecological health would be eager for specialist review at short notice. It is likely that the population referred to the paediatric gynaecology clinic and the cohort recruited in the follow-up study was biased towards more extensive adhesions, higher rates of parental anxiety, as well as concurrent diagnoses such as UTI, vulvovaginitis or urinary stream disturbances compared to the general population. Combining this with the younger median age in the follow-up cohort, it is likely that our results overestimate the rate of symptoms in girls with labial adhesions and underestimate the rate of spontaneous resolution.

Overall, parents had been reassured by their advice and were satisfied with expectant management, reflected in the low number who sought further review. Although UTIs and vulvovaginitis are often thought to be caused by labial adhesions, this relationship remains unclear as the etiology for both may be the same. Never the less, all girls in this cohort with urogenital symptoms settled with standard treatment, despite the conservative management of labial adhesions. The simple strategies given to girls with post-void dribbling were adequate as they did not return to clinic or seek further medical attention.

Given the above findings, treatment by un-anaesthetised manual separation of labial adhesions cannot be justified. The experiences of long-term distress caused by painful procedures in three of our patients suggest that the hazards of this procedure far outweigh the theoretical risks associated with conservative management. Our findings contradict the argument that there are no long-term consequences of manual separation of labial adhesions performed in young children.

Some questions remain regarding the timing of resolution of adhesions. This may be 
influenced by factors including hormonal levels and body mass and were not addressed in this study. What does appear clear is that provision of adequate advice to parents regarding the natural history of labial adhesions and the likelihood of resolution of minor urogenital symptoms with simple measures, allows a conservative approach to management with very low rates of parental concern and unnecessary intervention. Despite the very limited number of survey responses in the older age group, the absence of labial adhesions in clinical practice in the adolescent and adult gynaecology population supports the natural history of spontaneous resolution of adhesions.

We are reassured that the conservative approach advocated by the Gynaecology department is effective in allaying parents' concerns and preventing excessive treatments which are often ineffective and may be potentially harmful.

\section{References}

1. Bacon JL. Prepubertal labial adhesions: evaluation of a referral population. Am. J. Obstet. Gynecol. 2002; 187: 327-32.

2. Goldman RD. Child health update: estrogen cream for labial adhesion in girls. Can. Fam. Physician 2013; 59: 37-8.

3. Bacon JL, Romano ME, Quint EH. Clinical Recommendation: Labial Adhesions. J. Pediatr. Adolescent. Gynecol. 2015; 28: 405-9.

4. Van Eyk N, Allen L, Giesbrecht E, Jamieson MA, Kives S, Morris M, et al. Pediatric vulvovaginal disorders: a diagnostic approach and review of the literature. $J$. Obstet Gynaecol. Can. 2009; 31: 850-62.

5. Omar HA. Management of labial adhesions in prepubertal girls. J. Pediatr. Adolescent. Gynecol. 2000; 13: 183-5.

6. Bussen S, Eckert A, Schmidt U, Sutterlin M. Comparison of Conservative and Surgical Therapy Concepts for Synechia of the Labia in Pre-Pubertal Girls. Geburtshilfe Frauenheilkd. 2016; 76: 390-5. 
7. Watanabe T, Matsubara S, Fujinaga Y, Asada K, Ohmaru T, Suzuki M. Manual separation followed by local cleanliness for pediatric labial adhesion. J. Obstet. Gynaecol. Res. 2010; 36: 667-70.

8. Soyer T. Topical estrogen therapy in labial adhesions in children: therapeutic or prophylactic? J. Pediatr. Adolescent. Gynecol. 2007; 20: 241-4.

9. Granada C, Sokkary N, Sangi-Haghpeykar H, Dietrich JE. Labial adhesions and outcomes of office management. J. Pediatr. Adolescent. Gynecol. 2015; 28:109-13. 10. Mayoglou L, Dulabon L, Martin-Alguacil N, Pfaff D, Schober J. Success of treatment modalities for labial fusion: a retrospective evaluation of topical and surgical treatments. J. Pediatr. Adolescent. Gynecol. 2009; 22: 247-50.

11. Eroglu E, Yip M, Oktar T, Kayiran SM, Mocan H. How should we treat prepubertal labial adhesions? Retrospective comparison of topical treatments: estrogen only, betamethasone only, and combination estrogen and betamethasone. J. Pediatr. Adolescent. Gynecol. 2011; 24: 389-91.

12. Leung AK, Robson WL, Tay-Uyboco J. The incidence of labial fusion in children. J.Paediatr. Child Health. 1993; 29: 235-6.

13. Muram D. Treatment of prepubertal girls with labial adhesions. J. Pediatr. Adolescent. Gynecol. 1999; 12: 67-70.

14. Seehusen DA, Earwood JS. Postpartum labial adhesions. J. Am. Board Fam. Med. 2007; 20: 408-10.

15. Imamura R, Fujimoto M, Meguro N, Maeda O, Saiki S, Kinouchi T, et al. [Labial adhesion presenting as urinary incontinence and dysuria in a postmenopausal woman: a case report]. Hinyokika Kiyo. 1998; 44: 843-5.

16. Bradford J, Fischer G. Surgical division of labial adhesions in vulvar lichen sclerosus and lichen planus. J. Low. Genit. Tract Dis. 2013; 17: 48-50.

17. Garden AS. Vulvovaginitis and other common childhood gynaecological conditions. Arch. Dis. Child. Educ. Pract. Ed. 2011; 96: 73-8.

This article is protected by copyright. All rights reserved. 
18. Hutson JM, O'Brien M, Beasley SW, Teague WJ, King SK eds. Jones' Clinical Paediatric Surgery. Wiley Blackwell, 2015;164-171.

19. McCann J, Wells R, Simon M, Voris J. Genital findings in prepubertal girls selected for nonabuse: a descriptive study. Pediatrics. 1990; 86: 428-39.

20. Berenson AB, Heger AH, Hayes JM, Bailey RK, Emans SJ. Appearance of the hymen in prepubertal girls. Pediatrics. 1992; 89: 387-94.

21. Leung AK, Robson WL. Labial fusion and urinary tract infection. Child Nephrol. Urol. 1992; 12: 62-4.

22. Ben-Ami T, Boichis H, Hertz M. Fused labia. Clinical and radiological findings. Pediatr. Radiol. 1978; 7: 33-5.

23. Leung AKC, Robson WLM, Kao CP, Liu EKH, Fong JHS. Treatment of labial fusion with topical estrogen therapy. Clin. Pediatr. 2005; 44: 245-7.

24. Nurzia MJ, Eickhorst KM, Ankem MK, Barone JG. The surgical treatment of labial adhesions in pre-pubertal girls. J. Pediatr. Adolescent. Gynecol. 2003;16: 21-3.

25. Khanam W, Chogtu L, Mir Z, Shawl F. Adhesion of the labia minora-a study of 75 cases. Obstet Gynecol. Surv. 1978; 33: 364-5.

26. Thibaud E, Duflos C. [Plea for child: labial agglutination should not be treated]. Plaidoyer pour l'enfant: le traitement de la coalescence des petites levres est inutile. Arch Pediatr. 2003;10: 465-6.

27. Jenkinson SD, MacKinnon AE. Spontaneous separation of fused labia minora in prepubertal girls. B.M.J.(Clinical research ed). 1984; 289:160-1.

This article is protected by copyright. All rights reserved. 
Case 1: Now 24 years old, she described the experience of having her adhesions manually separated as follows: "I can only just remember sort of what happened, I just remember feeling uncomfortable and uneasy about what was happening."

Case 2: The mother of this girl took her daughter, age $\sim 12$ months, to her general practitioner (GP) after discovering her daughter's labial adhesions. The GP told the parents that the condition was easily treated and asked the father to hold his daughter whilst she separated the adhesions using a cotton-tip. Both parents were very distressed by the procedure and felt considerable guilt afterwards for the pain and distress they felt they had caused their child. The child's mother's memories of her own sexual assault were triggered by witnessing the procedure and she required psychological counselling as a consequence. The parents could not change their daughter's nappy for the next 6 months without causing their daughter considerable distress. The adhesions recurred soon after the procedure and the mother brought her daughter to $\mathrm{RCH}$ for a second opinion. At her initial appointment at $\mathrm{RCH}$ (age 20 months), the girl could not be examined due to distress. However, the mother was reassured by the advice she received and the adhesions were not treated again.

Case 3: The mother of this girl took her daughter to the GP at approximately 18 months, after discovering her adhesions. The GP applied a local anaesthetic gel and cut the adhesions with a scalpel while the mother was asked to hold her daughter. The patient is now 8 years old and still asks her mother why the doctor did that to her. Her mother reports that her daughter still remembers the doctor's 'long nails'. The mother remembers her daughter 'screaming for hours' afterwards and describes the procedure as traumatic for them both. The adhesions returned soon after and the mother attended RCH for a second opinion. She was happy to leave the adhesions untreated and was reassured by the advice she received.

Box 1. Patient accounts of the distress associated with lateral traction or surgical separation. 
Figure I Flowchart of patients identified with labial adhesions, outcomes and participation in the followup study

Figure 2 Age distribution of original cohort of girls with labial adhesions and those participating in the follow-up cohort in February 2017.

Figure 3 Distribution of patients according to age and whether adhesions had resolved at follow-up.

Figure 4 Each line shows the change in the extent of labial adhesions between patients' initial appointment and at the time of follow-up.

This article is protected by copyright. All rights reserved. 


\section{University Library}

\section{- M M N E R VA A gateway to Melbourne's research publications}

Minerva Access is the Institutional Repository of The University of Melbourne

Author/s:

Norris, JE;Elder, CV;Dunford, AM;Rampal, D;Cheung, C;Grover, SR

Title:

Spontaneous resolution of labial adhesions in pre-pubertal girls

Date:

2018-07-01

Citation:

Norris, J. E., Elder, C. V., Dunford, A. M., Rampal, D., Cheung, C. \& Grover, S. R.

(2018). Spontaneous resolution of labial adhesions in pre-pubertal girls. JOURNAL OF

PAEDIATRICS AND CHILD HEALTH, 54 (7), pp.748-753. https://doi.org/10.1111/jpc.13847.

Persistent Link:

http://hdl.handle.net/11343/283619 